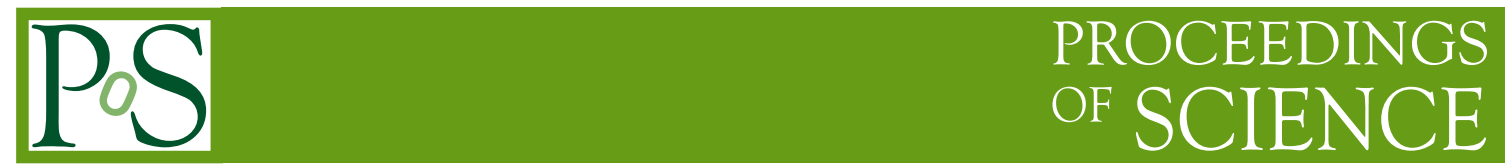

\title{
Recent results on Dark Matter
}

\author{
R. Bernabei ${ }^{1}$, P. Belli*1, F. Cappella ${ }^{2}$, R. Cerulli ${ }^{3}$, C.J. Dai ${ }^{4}$, A. d'Angelo ${ }^{2}$, H.L. He ${ }^{4}$, A. \\ Incicchitti $^{2}$, X.H. Ma ${ }^{4}$, F. Montecchia ${ }^{1,5}$, F. Nozzoli ${ }^{1}$, D. Prosperi ${ }^{2, * *}$, X.D. Sheng ${ }^{4}$, R.G. \\ Wang $^{4}$, Z.P. Ye ${ }^{4,6}$ \\ ${ }^{1}$ Dip. Fisica, Università di Roma "Tor Vergata" and INFN, sez. Roma Tor Vergata, Rome, Italy \\ ${ }^{2}$ Dip. Fisica, Università di Roma "La Sapienza" and INFN, sez. Roma, Rome, Italy \\ ${ }^{3}$ Laboratori Nazionali del Gran Sasso, I.N.F.N., Assergi, Italy \\ ${ }^{4}$ IHEP, Chinese Academy, P.O. Box 918/3, Beijing, China \\ ${ }^{5}$ Lab. Sperim. Policentrico di Ingegneria Medica, Università di Roma "Tor Vergata" \\ ${ }^{6}$ University of Jing Gangshan, Jiangxi, China \\ ** deceased
}

Experimental observations and theoretical considerations have shown that a large part of the Universe is made of Dark Matter particles; this has currently motivated experimental efforts aiming to investigate them by direct and indirect detection approaches. In particular, several techniques are developed giving just model-dependent results. The approach able indeed to offer a suitable model-independent signature is shortly described here and the obtained results are summarized.

The Xth Nicola Cabibbo International Conference on Heavy Quarks and Leptons,

October 11-15, 2010

Frascati (Rome) Italy

${ }^{*}$ Speaker. 


\section{Introduction}

The problem of the existence of Dark Matter (DM) in our Universe dates back to the astrophysical observations at the beginning of the past century. Many observations from the galactic to the cosmological scale support that most of the Universe is dark and large space for Dark Matter particles in the Universe exists. Most of the DM particles, relics from the Big Bang, were either produced at rest or non relativistic at decoupling time. The DM candidates have to be neutral, stable or quasi-stable (e.g. with a decay time of order of or larger than the age of the Universe) and have to be elusive in the interaction with ordinary matter. These features are respected, for example, by the axions, by axion-like candidates with mass in the $\mathrm{keV}$ range, by possible sterile neutrino, by a class of various kinds of candidates generically mentioned as WIMPs (Weakly Interacting Massive Particles) although they can have well different phenomenologies, and by many other possible candidates as extensively discussed in literature; other possibilities are open as well.

In the world several experiments aim to perform a direct DM investigation, using different kinds of detectors and exploiting different approaches; generally they can give just modeldependent results.

The DAMA/LIBRA and the previous DAMA/NaI experiments have indeed obtained an evidence for the presence of DM particles in the galactic halo with a cumulative $8.9 \sigma$ C.L. by exploiting the DM model-independent annual modulation signature.

\section{The DAMA/LIBRA experiment}

DAMA is an observatory for rare processes located deep underground at the Gran Sasso National Laboratory of the I.N.F.N.. It is based on the development and use of low background scintillators. Several set-ups have been realized and are in operation to investigate many rare processes $[1,2,3,4,5,6,7,8,9,10,11,12,13,14,15,16]$.

In particular, DAMA/LIBRA is investigating the presence of DM particles in the galactic halo by exploiting the model independent DM annual modulation signature [17]. In fact, as a consequence of its annual revolution around the Sun, which is moving in the Galaxy traveling with respect to the Local Standard of Rest towards the star Vega near the constellation of Hercules, the Earth should be crossed by a larger flux of Dark Matter particles around $\sim 2$ June (when the Earth orbital velocity is summed to the one of the solar system with respect to the Galaxy) and by a smaller one around $\sim 2$ December (when the two velocities are subtracted). This signature offers an efficient model independent signature, able to test a large number of DM candidates, a large interval of cross sections and of halo densities. The DM annual modulation signature is very distinctive since the corresponding signal must simultaneously satisfy all the following requirements: the rate must contain a component modulated according to a cosine function (1) with one year period (2) and a phase that peaks roughly around $\simeq 2^{\text {nd }}$ June (3); this modulation must only be found in a well-defined low energy range, where DM particle induced events can be present (4); it must apply only to those events in which just one detector of many actually "fires" (single-hit events), since the DM particle multi-interaction probability is negligible (5); the modulation amplitude in the region of maximal sensitivity must be $\lesssim 7 \%$ for usually adopted halo distributions (6), but it can be larger in case of some possible scenarios such as e.g. those in refs. [18, 19]. Only systematic effects 
or side reactions able to simultaneously fulfil all these requirements and to account for the whole observed modulation amplitude could mimic this signature; thus, no other effect investigated so far in the field of rare processes offers a so stringent and unambiguous signature.

In the following, we will just briefly summarize just some aspects of the results on the DM particle investigation obtained by DAMA/LIBRA, exploiting over six annual cycles the model independent DM annual modulation signature (exposure: 0.87 ton $\times$ yr) $[15,20]$. The data have also been combined with those previously collected by DAMA/NaI over 7 annual cycles $(0.29$ ton $\times$ yr). Thus, the total exposure collected over 13 annual cycles is 1.17 ton $\times y r$, this is orders of magnitude larger than the exposures typically released in the field.

The DAMA/NaI set up and its performances are reported in ref.[1, 3, 4, 5], while the DAMA/LIBRA set-up and its performances are described in ref. [14]. Here we just summarized the main features: i) the sensitive part of the set-up is made of 25 highly radiopure $\mathrm{NaI}(\mathrm{Tl})$ crystal scintillators placed in a 5-rows by 5-columns matrix; ii) the detectors' responses range from 5.5 to 7.5 photoelectrons $/ \mathrm{keV}$; iii) the hardware threshold of each PMT is at single photoelectron (each detector is equipped with two low background photomultipliers working in coincidence); iv) energy calibration with $\mathrm{X}$-rays $/ \gamma$ sources are regularly carried out down to few $\mathrm{keV}$ in the same conditions as the production runs; v) the software energy threshold of the experiment is $2 \mathrm{keV}$ (electron equivalent).

\section{$2-6 \mathrm{keV}$}

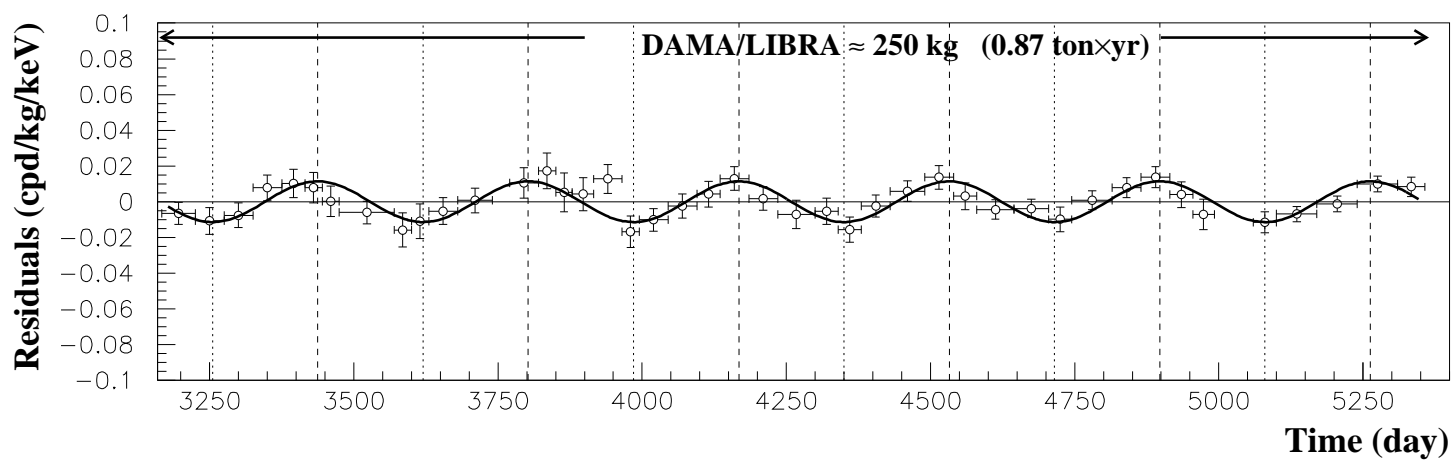

Figure 1: Experimental model-independent residual rate of the single-hit scintillation events, measured by DAMA/LIBRA, 1,2,3,4,5,6 in the $(2-6) \mathrm{keV}$ energy intervals as a function of the time. The experimental points present the errors as vertical bars and the associated time bin width as horizontal bars. The superimposed curves are the cosinusoidal functions behaviors $A \cos \omega\left(t-t_{0}\right)$ with a period $T=\frac{2 \pi}{\omega}=1 \mathrm{yr}$, with a phase $t_{0}=152.5$ day (June $2^{\text {nd }}$ ) and with modulation amplitudes, $A$, equal to the central values obtained by best fit over the whole data including also the exposure previously collected by the former DAMA/NaI experiment $(1.17$ ton $\times \mathrm{yr})$ The dashed vertical lines correspond to the maximum expected for the DM signal (June $2^{\text {nd }}$ ), while the dotted vertical lines correspond to the minimum. For details see [20] and refs. therein.

Several analyses on the model-independent DM annual modulation signature have been performed (see ref. [15, 20] and references therein); here just few arguments are mentioned. In particular, Fig. 1 shows the time behaviour of the experimental residual rates of the single-hit events collected by DAMA/LIBRA in the (2-6) keV energy interval $[15,20]$. The superimposed curve is the cosinusoidal function: $A \cos \omega\left(t-t_{0}\right)$ with a period $T=\frac{2 \pi}{\omega}=1 \mathrm{yr}$ and with a phase $t_{0}=152.5$ day (June $2^{\text {nd }}$ ), and modulation amplitude, $A$, obtained by best fit over the seven cycles of DAMA/NaI $[4,5]$ and the six of DAMA/LIBRA $[15,20]$. When the period and the phase pa- 
rameters are also released in the fit, values well compatible with those expected for a DM particle induced effect are obtained for the cumulative exposure [20] in the cumulative (2-6) keV energy interval: $A=(0.0116 \pm 0.0013) \mathrm{cpd} / \mathrm{kg} / \mathrm{keV}, T=(0.999 \pm 0.002) \mathrm{yr}$ and $t_{0}=(146 \pm 7)$ day. Summarizing, the analysis of the single-hit residual rate favours the presence of a modulated cosine-like behaviour with proper features at $8.9 \sigma$ C.L. [20].

The same data of Fig. 1 have also been investigated by a Fourier analysis, obtaining a clear peak corresponding to a period of 1 year [20]; this analysis in other energy region shows instead only aliasing peaks. Moreover, while in the (2-6) keV single-hit residuals a clear modulation is present, it is absent at energies just above [20]. The measured energy distribution has been investigated in other energy regions not of interest for Dark Matter, also verifying the absence of any significant background modulation. In fact, the background in the lowest energy region is essentially due to "Compton" electrons, X-rays and/or Auger electrons, muon induced events, etc., which are strictly correlated with the events in the higher energy part of the spectrum; thus, if a modulation detected in the lowest energy region would be due to a modulation of the background (rather than to a signal), an equal or larger modulation in the higher energy regions should be present. The data analyses have allowed to exclude the presence of a background modulation in the whole energy spectrum at a level much lower than the effect found in the lowest energy region for the single-hit events [20].

A further relevant investigation has been done by applying the same hardware and software procedures, used to acquire and to analyse the single-hit residual rate, to the multiple-hits one. In fact, since the probability that a DM particle interacts in more than one detector is negligible, a DM signal can be present just in the single-hit residual rate. Thus, this allows the test of the background behaviour in the same energy interval of the observed positive effect. A clear modulation is present in the single-hit events, while the fitted modulation amplitude of the multiple-hits residual rate is well compatible with zero [20]. Similar results were previously obtained also for the DAMA/NaI case [5]. Thus, again evidence of annual modulation with proper features, as required by the DM annual modulation signature, is present in the single-hit residuals (events class to which the DM particle induced events belong), while it is absent in the multiple-hits residual rate (event class to which only background events belong). Since the same identical hardware and the same identical software procedures have been used to analyse the two classes of events, the obtained result offers an additional strong support for the presence of DM particles in the galactic halo further excluding any side effect either from hardware or from software procedures or from background.

The annual modulation present at low energy has also been analyzed by depicting the differential modulation amplitudes, $S_{m, k}$, as a function of the energy (the $k$ index identifies the energy interval); the $S_{m, k}$ is the modulation amplitude of the modulated part of the signal obtained by maximum likelihood method over the data, considering $T=1 \mathrm{yr}$ and $t_{0}=152.5$ day. The $S_{m, k}$ values are reported as function of the energy in Fig. 2 and in refs. [20] (the $k$ index is omitted there for simplicity). It has been also verified that the measured modulation amplitudes are statistically well distributed in all the crystals, in all the annual cycles and energy bins; these and other discussions can be found in ref. [20].

Both the data of DAMA/LIBRA and of DAMA/NaI fulfil all the requirements of the DM annual modulation signature.

As previously done for DAMA/NaI $[4,5]$, careful investigations on absence of any signifi- 


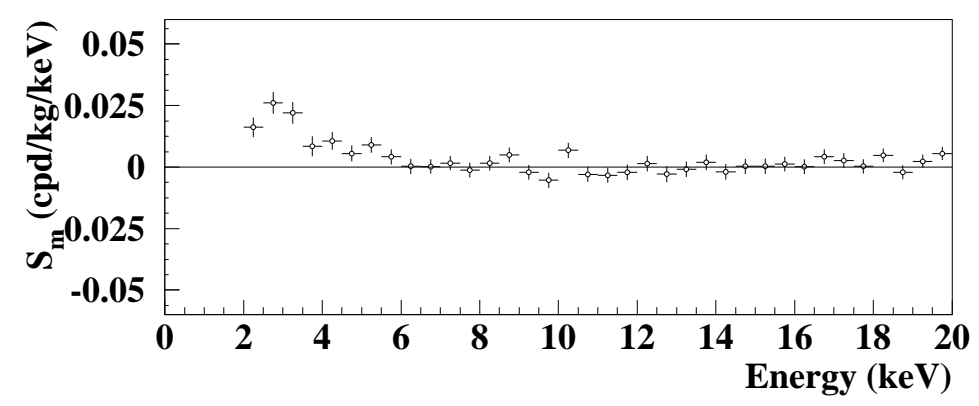

Figure 2: Energy distribution of the $S_{m}$ variable for the total cumulative exposure 1.17 ton $\times$ yr. The energy bin is $0.5 \mathrm{keV}$. A clear modulation is present in the lowest energy region, while $S_{m}$ values compatible with zero are present just above. In fact, the $S_{m}$ values in the (6-20) keV energy interval have random fluctuations around zero with $\chi^{2}$ equal to 27.5 for 28 degrees of freedom. See refs. [15, 20].

cant systematics or side reaction effect in DAMA/LIBRA have been quantitatively carried out and reported in details in ref. $[15,20]$ and refs. therein; some additional arguments have also been addressed in ref[21]. No systematics or side reactions able to mimic the signature (that is, able to account for the measured modulation amplitude and simultaneously satisfy all the requirements of the signature) has been found or suggested by anyone over more than a decade. For detailed quantitative discussions on all the related topics and for results see ref. $[15,20]$ and refs. therein.

In conclusion, DAMA/LIBRA has confirmed the presence of an annual modulation satisfying all the requirements of the DM annual modulation signature, as previously pointed out by DAMA/NaI; in particular, the evidence for the presence of DM particles in the galactic halo is cumulatively supported at $8.9 \sigma$ C.L..

As regards the corollary investigation on the nature of the DM candidate particle(s) and related astrophysical, nuclear and particle physics scenarios, it has been shown that the obtained model independent evidence can be compatible with a wide set of possibilities; see just as examples $[2,4,5,6,7,8,9,10,11,15]$. Many other interpretations of the annual modulation results are available in literature (as e.g. [18, 22], etc.); others are open.

\section{Comments and comparisons}

It is worth noting that no other experiment exists, whose result can be directly compared in a model-independent way with those by DAMA/NaI and DAMA/LIBRA, and that - more in general - results obtained with different target materials and/or different approaches cannot be directly compared among them in a model-independent way. This is in particular due to the existing experimental and theoretical uncertainties, not last e.g. how many kinds of dark matter particles can exist in the Universe, the nature, the interaction types, the different nuclear and/or atomic correlated aspects, the unknown right halo model, the right DM density, etc. as well as the uncertainties on the values of each one of the many involved experimental and theoretical parameter/assumption/approximation used in the calculations. Moreover, some experimental aspects of some techniques used in the field have also to be addressed [4, 23]. Another relevant argument is the methodological robustness [24]. In particular, the general considerations on comparisons reported in Appendix A of ref. [15] still hold. Hence, claims for contradiction have no scientific 
basis. On the other hand, whatever possible positive result has to be interpreted and a large room of compatibility with DAMA annual modulation evidence is present. Just as examples we remind some recent model dependent interpretations of results by CDMS-II, CoGeNT, CRESST [25]; see e.g. [26] and other literature.

Similar considerations can also be done for the indirect detection searches, since it does not exist a biunivocal correspondence between the observables in the direct and indirect experiments. However, if possible excesses in the positron to electron flux ratio and in the $\gamma$ rays flux with respect to some modeling of the background contribution, which is expected from the considered sources, might be interpreted - under some assumptions - in terms of Dark Matter, this would also be not in conflict with the effect observed by DAMA experiments. However, it is worth noting that different possibilities either considering different background modeling or accounting for other kinds of sources can also explain the indirect observations [27].

Finally, considering the relevance to lower the software energy threshold of the experiment, in order to improve the performance and the sensitivity of the experiment and to allow a highly precise determination of all the DM modulation parameters and deeper corollary information on the nature of the DM particle(s) and on the various related astrophysical, nuclear and particle Physics scenarios, the replacement of all the PMTs with new ones with higher quantum efficiency has been planned and work is in progress. DAMA/LIBRA will study second order effects as well, and several other rare processes as previously the former DAMA/NaI apparatus [12] and itself so far [16] have done.

\section{References}

[1] R. Bernabei et al., Il Nuovo Cim. A112, 545-576 (1999).

[2] R. Bernabei et al., Phys. Lett. B389, 757-766 (1996); R. Bernabei et al., Phys. Lett. B424, 195-201 (1998); R. Bernabei et al., Phys. Lett. B450, $448-455$ (1999); P. Belli et al., Phys. Rev. D61, 023512-9 (2000); R. Bernabei et al., Phys. Lett. B480, 23-31 (2000); R. Bernabei et al., Phys. Lett. B509, 197-203 (2001); R. Bernabei et al., Eur. Phys. J. C23, 61-64 (2002); P. Belli et al., Phys. Rev. D66, 043503-17 (2002).

[3] R. Bernabei et al., Eur. Phys. J. C18, 283-292 (2000).

[4] R. Bernabei el al., La Rivista del Nuovo Cimento 26 n.1, 1-73 (2003).

[5] R. Bernabei et al., Int. J. Mod. Phys. D13, 2127-2159 (2004).

[6] R. Bernabei et al., Int. J. Mod. Phys. A21, 1445-1469 (2006).

[7] R. Bernabei et al., Eur. Phys. J. C47, 263-271(2006).

[8] R. Bernabei et al., Int. J. Mod. Phys. A22, 3155-3168 (2007).

[9] R. Bernabei et al., Eur. Phys. J. C53, 205-213 (2008).

[10] R. Bernabei et al., Phys. Rev. D77, 023506-9 (2008).

[11] R. Bernabei et al., Mod. Phys. Lett. A23, 2125-2140 (2008).

[12] R. Bernabei et al., Phys. Lett. B408, 439-444 (1997); P. Belli et al., Phys. Lett. B460, 236-241 (1999); R. Bernabei et al., Phys. Rev. Lett. 83, 4918-4921 (1999); P. Belli et al., Phys. Rev. C60, 065501-7 (1999); R. Bernabei et al., Il Nuovo Cimento A112, 1541-1552 (1999); R. Bernabei et al., 
Phys. Lett. B515, 6-12 (2001); F. Cappella et al., Eur. Phys. J.-direct C14, 1-6 (2002); R. Bernabei et al., Eur. Phys. J. A23, 7-10 (2005); R. Bernabei et al., Eur. Phys. J. A24, 51-56 (2005); R. Bernabei et al., Astrop. Phys. 4, 45-54 (1995); R. Bernabei, in The identification of Dark Matter, World Sc. Pub., Singapore, 1997, pp. 574.

[13] DAMA web page: http://people.roma2.infn.it/dama

[14] R. Bernabei et al., Nucl. Instr. \& Meth. A592, 297-315 (2008).

[15] R. Bernabei et al., Eur. Phys. J. C56, 333-355 (2008).

[16] R. Bernabei et al., Eur. Phys. J. C62, 327-332 (2009).

[17] K. A. Drukier et al., Phys. Rev. D33, 3495-3508 (1986); K. Freese et al., Phys. Rev. D37, 3388-3405 (1988).

[18] D. Smith and N. Weiner, Phys. Rev. D 64 (2001) 043502; D. Tucker-Smith and N. Weiner, Phys. Rev. D 72 (2005) 063509; D. P. Finkbeiner et al, Phys. Rev. D 80 (2009) 115008.

[19] K. Freese et al., Phys. Rev. D71, 043516-15 (2005); Phys. Rev. Lett. 92, 111301-4 (2004).

[20] R. Bernabei et al., Eur. Phys. J. C67 (2010) 39.

[21] R. Bernabei et al., AIP Conf. Proceed. 1223 (2010) 50, arXiv:0912.0660[astro-ph.GA]; R. Bernabei et. al, J. Phys.: Conf. Ser. 203, 012040 (2010) (arXiv:0912.4200); http://taup2009.lngs.infn.it/slides/jul3/nozzoli.pdf, talk given by F. Nozzoli. R. Bernabei et. al, arXiv:1007.0595

[22] A. Bottino, N. Fornengo, and S. Scopel, Phys. Rev. D 67 (2003) 063519; A. Bottino, F. Donato, N. Fornengo, and S. Scopel, Phys. Rev. D 69 (2003) 037302; Phys. Rev. D 78 (2008) 083520; A. Bottino, F. Donato, N. Fornengo, S. Scopel, arXiv:0912.4025; R. Foot, Phys. Rev. D 78 (2008) 043529; Y. Bai and P.J. Fox, arXiv:0909.2900; K. Belotsky, D. Fargion, M. Khlopov and R.V. Konoplich, Phys. Atom. Nucl.71 (2008) 147; E.M. Drobyshevski et al., Astrohys. \& Astronom. Trans. 26:4 (2007) 289; Mod. Phys. Lett. A 23 (2008) 3077; Nima Arkani-Hamed et al., Phys. Rev. D79 (2009) 015014; Daniele S.M. Alves et al., arXiv:0903.3945; M. Khlopov et al., arXiv:1011.4587; Spencer Chang, Rafael F. Lang, Neal Weiner, arXiv:1007.2688; Spencer Chang, Neal Weiner, Itay Yavin, arXiv:1007.4200; Sarah Andreas, Andreas Ringwald, arXiv:1008.4519.

[23] R. Bernabei et al., Liquid Noble gases for Dark Matter searches: a synoptic survey, Exorma Ed., Roma, ISBN 978-88-95688-12-1, 2009, pp. 1-53 (arXiv:0806.0011v2); R. Bernabei et. al, J. Phys.: Conf. Ser. 203 (2010) 012040 (arXiv:0912.4200), http://taup2009.lngs.infn.it/slides/jul3/nozzoli.pdf, talk given by F. Nozzoli; J.I. Collar and D.N. McKinsey, arXiv:1005.0838; J.I. Collar and D.N. McKinsey, arXiv:1005.3723; J.I. Collar, arXiv:1006.2031.

[24] R. Hudson, Found. Phys. 39, 174-193 (2009).

[25] Z. Ahmed et al. (CDMS), Phys. Rev. Lett., 102 (2009) 011301; arXiv:0912.3592, C. E. Aalseth et al. [The CoGeNT Coll.], arXiv:1002.4703. See talk by W. Seidel (CRESST coll., WONDER 2010 Workshop, LNGS, Italy, March 22-23, 2010 and MPIK seminar by T. Schwetz, June 21, 2010.

[26] D. Hooper, Lisa Goodenough, arXiv:1010.2752; D. Hooper et al., arXiv:1007.1005; C. Kelso, D. Hooper, arXiv:1011.3076; D. Hooper et al., arXiv: arXiv:1011.4520; N. Fornengo et al., arXiv:1011.4743.

[27] F. Donato et al., Phys. rev. Lett. 102 (2009) 071301; T. Delahaye et al., Astron. Astrophys. 501 (2009) 821; S. Profumo, arXiv:0812.4457; P. Blasi, Phys. Rev. Lett. 103 (2009) 051104; M. Ahlers et al., arXiv:0909.4060. 